STOCHASTIC MODELS

Vol. 19, No. 1, pp. 125-147, 2003

\title{
Robust Positioning of Service Units
}

\author{
J. Puerto ${ }^{1, *}$ and A. M. Rodríguez-Chía ${ }^{2, *}$ \\ ${ }^{1}$ Dept. Estadística e Investigación Operativa, Universidad de Sevilla, \\ Fac. de Matemáticas, Sevilla, Spain \\ ${ }^{2}$ Dept. Estadística e Investigación Operativa, Universidad de Cádiz, Fac. de Ciencias \\ del Mar, Polígono Río San Pedro, Cádiz, Spain
}

\begin{abstract}
In this paper, we address the problem of locating mobile service units to cover random incidents. The model does not assume complete knowledge of the probability distribution of the location of the incident to be covered. Instead, only the mean value of that distribution is known. We propose the minimization of the maximum expected response time as an effectiveness measure for the model. Thus, the solution obtained is robust with respect to any probability distribution. The cases of one and two service units under the nearest allocation rule are studied in the paper. For both problems, the optimal solutions are shown to be degenerate distributions for the servers.
\end{abstract}

Key Words: Location theory; Stochastic problems.

\section{INTRODUCTION}

In distribution systems and in continuous location models, a common problem is to find the optimal placement of one or more servers minimizing the distances to a given set of points. All the models considered so far in the literature assume that the positions of

*Correspondence: J. Puerto, Dept. Estadística e Investigación Operativa, Universidad de Sevilla, Fac. de Matemáticas, C/Tarfia s/n, 41012 Sevilla, Spain; E-mail: puerto@cica.es. A. M. RodríguezChía, Dept. Estadística e Investigación Operativa, Universidad de Cádiz, Fac. de Ciencias del Mar, Polígono Río San Pedro, 11510 Puerto Real, Cádiz, Spain; E-mail: antonio.rodriguezchia@uca.es.

125

DOI: $10.1081 / \mathrm{STM}-120018142$

1532-6349 (Print); 1532-4214 (Online)

Copyright (C) 2003 by Marcel Dekker, Inc.

www.dekker.com 
these points are either deterministic or distributed according to a known probability distribution on the family of Borel sets in $\mathbb{R}^{n}$ (see for instance Anderson and Fontenot, ${ }^{[1]}$ Carrizosa, Muñoz-Márquez and Puerto, ${ }^{[2]}$ Larson and Odoni,${ }^{[5]}$ Levine,${ }^{[6]}$ De Palma, Liu and Thisse, ${ }^{[7]}$ among others).

However, it is easy to find situations in the real-world where the hypothesis of complete knowledge of this probability distribution is unrealistic. In this paper, we propose a more general model where only the mean value of this distribution is known. This assumption is not really restrictive because we can obtain good estimates of the unknown mean value by sampling. Although more information can be obtained from the sample, our model only needs the estimation of the mean value, which is a very wellsolved problem in mathematical statistics. A real-world application of such models is, for instance, the problem of locating a read/write head of a computer hard-disk to easily access the stored data. Similarly, our framework includes the problem of positioning police-cars that must cover incidents where the law is being broken, and positioning idle elevators to minimize response time(see Vickson, Gerchak and Rotem ${ }^{[10]}$ or Smith ${ }^{[9]}$ for a different analyses assuming that the distribution of the data is known). Indeed, in these cases, usually the distribution of the places where the law will be broken, the data are stored, or the elevator is needed is not known. Nevertheless, it would be less restrictive to assume that either we know the mean value for these distributions or we may estimate it by means of an empirical study.

When the probability distribution of the position of the incident is unknown, the classical minimization of the expected distances is not possible. Therefore, alternative approaches have to be considered. In this paper, we propose a robust alternative consisting of minimizing the maximum expected distance within the whole family of probability measures which model the incident (see Gallego ${ }^{[4]}$ and Puerto and Fernánde ${ }^{[8]}$ for similar analyses applied to different problems in Operations Research).

Let $\mathcal{F}(\lambda)$ and $\mathcal{G}(\mu)$ be the families of random variables (r.v.) (given by their cumulative distribution functions (c.d.f.) defined on the $n$-dimensional hypercube $[0,1]^{n}$ with mean values $\lambda \in \mathbb{R}^{n}$ for $\mathcal{F}(\lambda)$ and $\mu \in \mathbb{R}^{n}$ for $\mathcal{G}(\mu)$, that is,

$$
\begin{aligned}
& \mathcal{F}(\lambda)=\left\{X \text { : r.v. on }[0,1]^{n} \text { with c.d.f. } F_{X}, \int_{[0,1]^{n}} x d F_{X}(x)=\lambda\right\}, \\
& \mathcal{G}(\mu)=\left\{A: \text { r.v. on }[0,1]^{n} \text { with c.d.f. } G_{A}, \quad \int_{[0,1]^{n}} a d G_{A}(a)=\mu\right\} .
\end{aligned}
$$

Define

$$
\mathcal{F}:=\bigcup_{\lambda \in[0,1]^{n}} \mathcal{F}(\lambda) .
$$

The families $\mathcal{F}$ and $\mathcal{G}(\mu)$ are the sets of random variables which model the position of the server and the incident, respectively. It is worth noting that we have defined these random variables in the $n$-dimensional hypercube $[0,1]^{n}$, but they can be extended to any hyperrectangle by a linear transformation.

As previously mentioned, some authors have studied the problem of minimizing the expected distance to the random incident, i.e.,

$$
\min _{X \in \mathcal{F}} \int_{[0,1]^{n}} \int_{[0,1]^{n}} d(x, a) d G_{A}(a) d F_{X}(x),
$$


where $d$ is a measure of distance, $X$ is a r.v. with c.d.f. $F_{X}$, representing the position of the server, and $A$ is a r.v. with c.d.f. $G_{A}$, representing the position of the incident.

Our model does not assume any a priori knowledge about the probability distribution of the incident apart from its mean value. That is to say, we have almost complete uncertainty about where the incident will take place, and we search for the policy that an emergency unit, $X$, has to follow to minimize the maximum expected distance to any random incident. Therefore, the problem is

$$
\min _{X \in \mathcal{F}} \max _{A \in \mathcal{G}(\mu)} \int_{[0,1]^{n}} \int_{[0,1]^{n}}\|x-a\|_{1} d G_{A}(a) d F_{X}(x),
$$

where $\|\cdot\|_{1}$ is the $l_{1}$-norm in $\mathbb{R}^{n}$, so that, for $x=\left(x_{1}, \ldots, x_{n}\right) \in \mathbb{R}^{n}$ we have that

$$
\|x\|_{1}=\sum_{i=1}^{n}\left|x_{i}\right|
$$

The readers should note that there are essentially two kinds of factors that influence the formulation of the problem: 1 ) the dimension $n$ of the space where the incidents occur; and 2) the number of service units to be located.

It is also worth noting that this problem formulation can be used to model the above mentioned real-world situations because: a) we do not need to know the distribution of the incident; and b) the read/write head only admits displacements following the directions of the coordinate axes; and both the highway and the trajectory of the elevator can be considered like line segments where displacements are linear. Thus, the $l_{1}$-norm is an appropriate measure of distance.

Finally, the formulation (1) gives us a new interpretation of the solutions obtained in terms of statistics. As we shall show in the paper, the optimal probability distributions for our problem are degenerate random variables. Since principal points of probability distributions are those points optimizing some effectiveness measure (see Flury ${ }^{[3]}$ ), we can see our solutions as a generalization of the principal points, but now we are optimizing over a family of distributions with a fixed mean rather than the values of a single probability distribution.

The paper is organized as follows. In Section 2, we consider the problem of locating a single facility; we first study the problem considering the service unit as a degenerate random variable and then we extend these results to the general case with any random variable. In Section 3, we consider the two-facility problem under the nearest allocation rule and we follow a scheme similar to that followed in Section 2. In Section 4, we include some concluding remarks and possible extensions to the considered model. Finally, in the Appendix, we include, for the sake of readability, several technical results that have been used in the paper.

\section{THE SINGLE FACILITY PROBLEM}

We begin this section by considering the one-dimensional case, then we proceed to the $n$-dimensional single facility problem. Let $\mathcal{F}_{1}(\lambda)$ and $\mathcal{G}_{1}(\mu)$ be, respectively, the families of random variables $\mathcal{F}(\lambda)$ and $\mathcal{G}(\mu)$ in the 1-dimensional case. For ease of 
understanding, we distinguish two cases. In the first case, the server is not allowed to patrol, i.e., we model the location of the server with a degenerate random variable. In the second case, the server is allowed to patrol, which means that it is any random variable in $\mathcal{F}_{1}$. For the first case, the mathematical formulation of the problem is:

$$
\min _{x \in[0,1]} \max _{A \in \mathcal{G}_{1}(\mu)} \int_{[0,1]}|x-a| d G_{A}(a) .
$$

Theorem 2.1 The optimal positioning policy in the hypothesis of Problem (2) is

$$
x^{*}= \begin{cases}0 & \text { if } \mu=0.5 \\ y \text { for any } y \in[0,1] & \text { if } \mu=0.5 \\ 1 & \text { if } \mu>0.5\end{cases}
$$

Remark 2.1 This result states that the optimal location for a fixed service unit when only the mean value $\mu$ of the distribution of the incident is known, is on an extreme point of the interval of feasible locations for the incident. Further, when $\mu=0.5$ any point on the interval is an optimal location of the server.

Proof: By Lemma A.2 in the Appendix we have that

$$
\min _{x \in[0,1]} \max _{A \in \mathcal{G}_{1}(\mu)} \int_{[0,1]}|x-a| d G_{A}(a)=\min _{x \in[0,1]} \max _{A \in \mathcal{G}_{1}(\mu)}\left(2 \int_{0}^{x} G_{A}(a) d a-x+\mu\right) .
$$

Hence, we prove that the maximum in the last expression is reached at the random variable $A^{*}$ with the following c.d.f.

$$
G_{A^{*}}(a)= \begin{cases}0 & \text { if } a<0 \\ 1-\mu & \text { if } 0 \leq a<1 \\ 1 & \text { if } a \geq 1 .\end{cases}
$$

Indeed, since $x$ and $\mu$ are constants for the inner maximum in the right hand side of (4), we have to prove the following inequality

$$
\int_{0}^{x} G_{A}(a) d a-(1-\mu) x \leq 0, \quad \forall x \in[0,1], \quad \forall A \in \mathcal{G}_{1}(\mu) .
$$

But, since $\int_{0}^{1} G_{A}(a) d a=1-\mu\left(\right.$ Lemma A.2) and $G_{A}(\cdot)$ is a distribution function, we are under the hypotheses of Lemma A.1 which proves the inequality (5).

Therefore, the minimization Problem (2) reduces to the following problem:

$$
\min _{x \in[0,1]} x(1-2 \mu)+\mu .
$$

Hence, depending on the relative values of $\mu$, we obtain that the optimal positioning $x *$ satisfies equation (3). 
In the second case, the service unit is also allowed to patrol. Initially, we permit the service unit to be distributed on the interval according to a random variable with the only condition that its mean value is fixed to $\lambda$. Then, we solve the case when $\lambda$ is not fixed. For the first case, the problem is

$$
\min _{X \in \mathcal{F}_{1}(\lambda)} \max _{A \in \mathcal{G}_{1}(\mu)} \int_{[0,1]} \int_{[0,1]}|x-a| d G_{A}(a) d F_{X}(x) .
$$

Theorem 2.2 Any random variable $X \in \mathcal{F}_{1}(\lambda)$ constitutes an optimal policy for Problem (6).

Proof: By Lemma A.4, we have that

$$
\int_{[0,1]} \int_{[0,1]}|x-a| d G_{A}(a) d F_{X}(x)=2\left(1-\int_{0}^{1} G_{A}(y) F_{X}(y) d y\right)-\lambda-\mu .
$$

Therefore, using that $\lambda$ and $\mu$ are fixed,we can solve Problem (6) by solving the equivalent problem

$$
\max _{X \in \mathcal{F}_{1}(\lambda)} \min _{A \in \mathcal{G}_{1}(\mu)} \int_{0}^{1} G_{A}(y) F_{X}(y) d y .
$$

In order to do this, we are going to prove that the inner minimum is achieved by the random variable $A^{*}$ such that $P\left(A^{*}=0\right)=1-\mu$ and $P\left(A^{*}=1\right)=\mu$.

$$
I_{A, X}:=\int_{0}^{1} F_{X}(y) G_{A}(y) d y-\int_{0}^{1} F_{X}(y)(1-\mu) d y \geq 0 .
$$

Considering $t_{0}:=t_{0}(A) \in(0,1)$ such that $t_{0}=\inf \left\{t \in \mathbb{R}: G_{A}(t) \geq 1-\mu\right\}$ we have the following inequalities:

$$
\begin{aligned}
I_{A, X} & =\int_{0}^{t_{0}} F_{X}(y)\left(G_{A}(y)-(1-\mu)\right) d y+\int_{t_{0}}^{1} F_{X}(y)\left(G_{A}(y)-(1-\mu) d y\right. \\
& \geq \int_{0}^{t_{0}} F_{X}\left(t_{0}\right)\left(G_{A}(y)-(1-\mu)\right) d y+\int_{t_{0}}^{1} F_{X}\left(t_{0}\right)\left(G_{A}(y)-(1-\mu)\right) d y \\
& =F_{X}\left(t_{0}\right)\left(\int_{0}^{1}\left(G_{A}(y)-(1-\mu)\right) d y\right)=0,
\end{aligned}
$$

by Lemma A.2. Similarly, Lema A.2 implies that

$$
\begin{aligned}
\min _{A \in \mathcal{G}_{1}(\mu)} \int_{0}^{1} G_{A}(y) F_{X}(y) d y+\sum_{a \in(0,1)} a P[A=a] P[X=a] & =\int_{0}^{1} F_{X}(y)(1-\mu) d y \\
& =(1-\lambda)(1-\mu),
\end{aligned}
$$

regardless of the choice of $X \in \mathcal{F}_{1}(\lambda)$, and the result follows. 


\section{Puerto and Rodríguez-Chía}

Let us consider in the following that no assumptions are made on the mean value, $\lambda$, of the random variable modelling the service unit. In this situation, the problem is

$$
\min _{X \in \mathcal{F}_{1}} \max _{A \in \mathcal{G}_{1}(\mu)} \int_{[0,1]} \int_{[0,1]}|x-a| d G_{A}(a) d F_{X}(x) .
$$

Corollary 2.1 An optimal positioning policy of Problem (7) is the random variable $X^{*}$ such that $P\left[X^{*}=x^{*}\right]=1$ where $x^{*}$ was defined in (3).

Proof: Note that

$$
\begin{aligned}
& \min _{X \in \mathcal{F}_{1}} \max _{A \in \mathcal{G}_{1}(\mu)} \int_{[0,1]} \int_{[0,1]}|x-a| d G_{A}(a) d F_{X}(x) \\
& \quad=\min _{\lambda \in[0,1]} \min _{X \in \mathcal{F}_{1}(\lambda)} \max _{A \in \mathcal{G}_{1}(\mu)} \int_{[0,1]} \int_{[0,1]}|x-a| d G_{A}(a) d F_{X}(x) .
\end{aligned}
$$

Let $H(\lambda)=\min _{X \in \mathcal{F}_{1}(\lambda)} \max _{A \in \mathcal{G}_{1}(\mu)} \int_{[0,1]} \int_{[0,1]}|x-a| d G_{A}(a) d F_{X}(x)$.

For each $\lambda \in[0,1]$, by the proof of Theorem 2.2 we have that

$$
H(\lambda)=2(1-(1-\lambda)(1-\mu))-\lambda-\mu=(1-2 \mu) \lambda+\mu .
$$

Thus, if we look for the minimum in $\lambda$ we obtain

$$
\arg \min _{\lambda \in[0,1]} H(\lambda)= \begin{cases}\{0\} & \text { if } \mu<0.5 \\ y \text { for any } y \in[0,1] & \text { if } \mu=0.5 \\ \{1\} & \text { if } \mu>0.5\end{cases}
$$

and the result follows.

This corollary shows that it is optimal to park the service unit when no hypotheses are made on the distribution of the service unit and only the mean value of the incident is known. Thus, although patrolling may be good for other reasons such as crime prevention, etc., it is not necessary in order to minimize the maximum expected distance to any random incident.

Finally, we also solve the $n$-dimensional problem. Indeed, let us consider the problem:

$$
\min _{X \in \mathcal{F}} \max _{A \in \mathcal{G}(\mu)} \int_{[0,1]^{n}} \int_{[0,1]^{n}} \sum_{i=1}^{n}\left|x_{i}-a_{i}\right| d G_{A}(a) d F_{X}(x),
$$

where $\mu=\left(\mu_{1}, \ldots, \mu_{n}\right), x=\left(x_{1}, \ldots, x_{n}\right)$ and $a=\left(a_{1}, \ldots, a_{n}\right)$. Problem (8) can be written equivalently as follows:

$$
\begin{aligned}
& \min _{X \in \mathcal{F}} \max _{A \in \mathcal{G}(\mu)} \sum_{i=1}^{n} \int_{[0,1]} \int_{[0,1]}\left|x_{i}-a_{i}\right| d G_{A_{i}}\left(a_{i}\right) d F_{X_{i}}\left(x_{i}\right) \\
& \quad \leq \min _{X \in \mathcal{F}} \sum_{i=1}^{n} \max _{A_{i} \in \mathcal{G}_{1}\left(\mu_{i}\right)} \int_{[0,1]} \int_{[0,1]}\left|x_{i}-a_{i}\right| d G_{A_{i}}\left(a_{i}\right) d F_{X_{i}}\left(x_{i}\right),
\end{aligned}
$$


where $G_{A_{i}}$ and $F_{X_{i}}$ are the marginal distributions of $G_{A}$ and $F_{X}$ respectively.

Let $A_{1}^{*}, \ldots, A_{n}^{*}$ be the 1-dimensional random variables attaining the inner maxima and $G_{A_{1}^{*}}, \ldots, G_{A_{n}^{*}}$ their respective cumulative distribution functions. Consider $d G_{A^{*}}=$ $d G_{A_{1}^{*}} \times \ldots \times d G_{A_{n}^{*}}$, the measure in the product space generated by the measures $d G_{A_{1}^{*}}, \ldots, d G_{A_{n}^{*}}$, and let $A^{*}$ be a $n$-dimensional random variable with cumulative distribution $G_{A^{*}}$. That means, $A *$ is a random vector whose components are independent random variables. Since $A *$ is feasible for the former maximum in (8), we have that (9) holds with equality. By a similar argument, we get

$$
\begin{aligned}
& \min _{X \in \mathcal{F}} \max _{A \in \mathcal{G}(\mu)} \int_{[0,1]^{n}} \int_{[0,1]^{n^{n}}} \sum_{i=1}^{n}\left|x_{i}-a_{i}\right| d G_{A}(a) d F_{X}(x) \\
& =\sum_{i=1}^{n} \min _{X_{i} \in \mathcal{F}_{1}} \max _{A_{i} \in \mathcal{G}_{1}\left(\mu_{i}\right)} \int_{[0,1]} \int_{[0,1]}\left|x_{i}-a_{i}\right| d G_{A_{i}}\left(a_{i}\right) d F_{X_{i}}\left(x_{i}\right) .
\end{aligned}
$$

Thus, we have obtained that the $n$-dimensional problem can be solved by solving $n$ different 1-dimensional problems. This reduction allows the resolution of Problem (8) by Corollary 2.1. In particular, the results in this section show that if the $l_{1}$-norm is used, the optimal policy is to park (to fix) the service unit at some vertex of the region where the random incident takes place.

\section{THE TWO-FACILITY PROBLEM}

In the previous section, we considered the problem of locating only one facility to cover a random incident. However, often more than one service unit is necessary, especially if the coverage region is large. In this section, we consider the case where two service facilities cover a random incident under the usual nearest allocation rule: the random incident is covered by the closest service unit. This allocation rule leads to the following formulation:

$$
\min _{X_{1}, X_{2} \in \mathcal{F}_{1}} \max _{A \in \mathcal{G}_{1}(\mu)} \int_{[0,1]^{2}} \int_{[0,1]} \min \left\{\left|x_{1}-a\right|,\left|x_{2}-a\right|\right\} d G_{A}(a) d F_{1,2}\left(x_{1}, x_{2}\right),
$$

where $\mathcal{F}_{1}, \mathcal{G}_{1}(\mu)$ were defined in Section $2, G_{A}(\cdot)$ is the c.d.f. of the random variable $A$ and $F_{1,2}(\cdot$,$) is the joint c.d.f. of the random variables X_{1}$ and $X_{2}$. It is worth noting that this is a non-trivial problem: 1) it is a minmax problem, and 2) the decision space is a functional space of random vectors.

This formulation allows us to model different real-world situations where there are two-service units to cover a random incident. This is for example the case of highways with two patrolling vehicles so that each one covers the closest incident.

In order to solve this problem, first we consider the case where the servers are not allowed to patrol, that is, $X_{1}$ and $X_{2}$ are degenerate random variables. After that, we deal with the general case: $X_{1}$ and $X_{2}$ are any random variables belonging to $\mathcal{F}_{1}$. 
132

\section{Puerto and Rodríguez-Chía}

The formulation of Problem (10) for the first case is given by the following expression

$$
\min _{x_{1}, x_{2} \in[0,1]} \max _{A \in \mathcal{G}_{1}(\mu)} \int_{[0,1]} \min \left\{\left|x_{1}-a\right|,\left|x_{2}-a\right|\right\} d G_{A}(a) .
$$

Remark 3.1 Without loss of generality we can assume that $x_{1} \leq x_{2}$.

Before we proceed to obtain the solution of Problem (11), we define the following functions:

$$
\begin{aligned}
& \bar{d}\left(x_{1}, x_{2}, A\right):=2\left(\int_{0}^{x_{1}} G_{A}(a) d a+\int_{\frac{x_{1}+x_{2}}{2}}^{x_{2}} G_{A}(a) d a\right)+\mu-x_{2}, \\
& C\left(x_{1}, x_{2}, p_{1}\right):=2\left((1-\mu) \frac{x_{1}+x_{2}}{2}-p_{1}\left(x_{1}-\left(\frac{x_{1}+x_{2}}{2}\right)^{2}\right)\right)-x_{2}+\mu,
\end{aligned}
$$

and the set

$$
\begin{aligned}
& T\left(x_{1}, x_{2}\right):=\left\{p=\left(p_{0}, p_{1}, p_{2}\right) \geq 0: p_{0}+p_{1}+p_{2}=1\right. \\
& \left.\quad \text { and } p_{1} \frac{x_{1}+x_{2}}{2}+p_{2}=\mu\right\},
\end{aligned}
$$

where $A$ is a random variable in $\mathcal{G}_{1}(\mu)$ with distribution function $G_{A}$ and $x_{1}, x_{2} \in[0,1]$.

Theorem 3.1 The optimal positioning policy in the hypothesis of Problem (11) is $x_{1}=\mu^{2}$ and $x_{2}=2 \mu-\mu^{2}$.

Proof: First, by Lemma A.5 and A.7, we have that

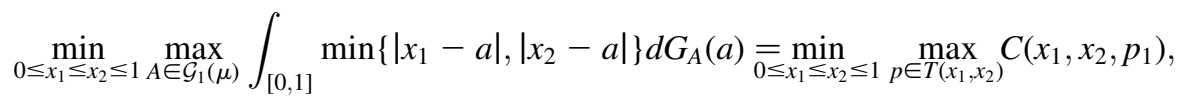

where $C$ and $T\left(x_{1}, x_{2}\right)$ were defined in (13) and (14), respectively. By Lemma A.8 the optimal solution of this problem is $x_{1}=\mu^{2}$ and $x_{2}=2 \mu-\mu^{2}$ and the proof is concluded.

Once we have studied the problem of locating two deterministic service units, we consider the general problem where the service units are random vectors. In this case we consider the original Problem (10).

Theorem 3.2 The optimal positioning policy of Problem (10) are the random variables $X_{1}^{*}$ and $X_{2}^{*}$ such that $P\left[X_{1}^{*}=\mu^{2}\right]=1$ and $P\left[X_{2}^{*}=2 \mu-\mu^{2}\right]=1$. 
Proof: Using Lemma A.5, we can bound the expression in (10) as follows (recall that $\bar{d}$ was defined in (12)):

$$
\begin{aligned}
& \max _{A \in \mathcal{G}_{1}(\mu)} \int_{[0,1]^{2}} \int_{[0,1]} \min \left\{\left|x_{1}-a\right|,\left|x_{2}-a\right|\right\} d G_{A}(a) d F_{1,2}\left(x_{1}, x_{2}\right) \\
& =\max _{A \in \mathcal{G}_{1}(\mu)}\left[\int_{\left[0, x_{2}\right] \times[0,1]} \bar{d}\left(x_{1}, x_{2}, A\right) d F_{1,2}\left(x_{1}, x_{2}\right)+\int_{\left(x_{2}, 1\right] \times[0,1]} \bar{d}\left(x_{2}, x_{1}, A\right) d F_{1,2}\left(x_{1}, x_{2}\right)\right] \\
& \leq \int_{\left[0, x_{2}\right] \times[0,1]} \max _{A \in \mathcal{G}_{1}(\mu)} \bar{d}\left(x_{1}, x_{2}, A\right) d F_{1,2}\left(x_{1}, x_{2}\right) \\
& \quad+\int_{\left(x_{2}, 1\right] \times[0,1]} \max _{A \in \mathcal{G}_{1}(\mu)} \bar{d}\left(x_{2}, x_{1}, A\right) d F_{1,2}\left(x_{1}, x_{2}\right) .
\end{aligned}
$$

Define

$$
\begin{aligned}
& S_{1}=\left\{\left(x_{1}, x_{2}\right) \in \mathbb{R}^{2}: 0 \leq x_{1} \leq x_{2} \leq 1\right\}, \\
& S_{2}=\left\{\left(x_{1}, x_{2}\right) \in \mathbb{R}^{2}: 0 \leq x_{2}<x_{1} \leq 1\right\},
\end{aligned}
$$

and let $\mathcal{X}_{S_{j}}(\cdot)$ denote the indicator function of the set $S_{j}$ for $j=1,2$. Now, Lemma A.7 allows to write the integrands in (15) as

$$
\begin{aligned}
& \max _{A \in \mathcal{G}_{1}(\mu)} \bar{d}\left(x_{1}, x_{2}, A\right)=\max _{p \in T\left(x_{1}, x_{2}\right)} C\left(x_{1}, x_{2}, p_{1}\right) \text { for }\left(x_{1}, x_{2}\right) \in S_{1}, \\
& \max _{A \in \mathcal{G}_{1}(\mu)} \bar{d}\left(x_{2}, x_{1}, A\right)=\max _{p \in T\left(x_{1}, x_{2}\right)} C\left(x_{2}, x_{1}, p_{1}\right) \text { for }\left(x_{1}, x_{2}\right) \in S_{2} .
\end{aligned}
$$

Combining (16) and (17) we can rewrite the expression (15) as

$$
\int_{[0,1]^{2}} \max _{p \in T\left(x_{1}, x_{2}\right)}\left[C\left(x_{1}, x_{2}, p_{1}\right) \mathcal{X}_{S_{1}}\left(x_{1}, x_{2}\right)+C\left(x_{2}, x_{1}, p_{1}\right) \mathcal{X}_{S_{2}}\left(x_{1}, x_{2}\right)\right] d F_{1,2}\left(x_{1}, x_{2}\right) .
$$

Let $p^{*}\left(x_{1}, x_{2}\right)=\left(p_{0}^{*}\left(x_{1}, x_{2}\right), p_{1}^{*}\left(x_{1}, x_{2}\right), p_{2}^{*}\left(x_{1}, x_{2}\right)\right) \in T\left(x_{1}, x_{2}\right)$ be the function where the expression above reaches its inner maximum. Notice that the expression of $p *\left(x_{1}, x_{2}\right)$ can be obtained from the proof of Lemma A.8, and it is defined in a different way depending on the region that $\left(x_{1}, x_{2}\right)$ belongs to.

Now, for all $\left(x_{1}, x_{2}\right) \in[0,1]^{2}$, let $A *\left(x_{1}, x_{2}\right)$ be a random variable independent of $\left(X_{1}, X_{2}\right)$, whose probability distribution is $\left.d G_{A *\left(x_{1}\right.}, x_{2}\right)$, defined by

$$
d G_{A^{*}\left(x_{1}, x_{2}\right)}(a)= \begin{cases}p_{0}^{*}\left(x_{1}, x_{2}\right) & \text { if } a=0 \\ p_{1}^{*}\left(x_{1}, x_{2}\right) & \text { if } a=\frac{x_{1}+x_{2}}{2} \\ p_{2}^{*}\left(x_{1}, x_{2}\right) & \text { if } a=1 .\end{cases}
$$

Notice that, for a fixed $x_{1}$ and $x_{2}$ belonging to the interval $[0,1], A *\left(x_{1}, x_{2}\right)$ is a discrete random variable taking the values $0, \frac{x_{1}+x_{2}}{2}$ and 1 with probabilities $p_{0}^{*}\left(x_{1}, x_{2}\right), p_{1}^{*}\left(x_{1}, x_{2}\right)$ and 
134

\section{Puerto and Rodríguez-Chía}

$p_{2}^{*}\left(x_{1}, x_{2}\right)$ respectively. Thus, by the definition of the functions $\bar{d}$ and $C$ (see the proof of Lemma A.7), $A *\left(x_{1}, x_{2}\right)$ verifies that

$$
\begin{aligned}
& \bar{d}\left(x_{1}, x_{2}, A^{*}\left(x_{1}, x_{2}\right)\right)=C\left(x_{1}, x_{2}, p_{1}^{*}\left(x_{1}, x_{2}\right)\right) \text { with }\left(x_{1}, x_{2}\right) \in S_{1}, \\
& \bar{d}\left(x_{2}, x_{1}, A^{*}\left(x_{1}, x_{2}\right)\right)=C\left(x_{2}, x_{1}, p_{1}^{*}\left(x_{1}, x_{2}\right)\right) \text { with }\left(x_{1}, x_{2}\right) \in S_{2} .
\end{aligned}
$$

Now, using (19), (20), and Lemma A.5, we have that

$$
\begin{aligned}
& \int_{[0,1]^{2}} \max _{p \in T\left(x_{1}, x_{2}\right)}\left[C\left(x_{1}, x_{2}, p_{1}\right) \mathcal{X}_{S_{1}}\left(x_{1}, x_{2}\right)+C\left(x_{2}, x_{1}, p_{1}\right) \mathcal{X}_{S_{2}}\left(x_{1}, x_{2}\right)\right] d F_{1,2}\left(x_{1}, x_{2}\right) \\
& =\int_{[0,1]^{2}}\left[C \left(x_{1}, x_{2}, p_{1}^{*}\left(x_{1}, x_{2}\right) \mathcal{X}_{S_{1}}\left(x_{1}, x_{2}\right)\right.\right. \\
& \left.\quad+C\left(x_{2}, x_{1}, p_{1}^{*}\left(x_{1}, x_{2}\right)\right) \mathcal{X}_{S_{2}}\left(x_{1}, x_{2}\right)\right] d F_{1,2}\left(x_{1}, x_{2}\right) \\
& =\int_{\left[0, x_{2}\right] \times[0,1]} \bar{d}\left(x_{1}, x_{2}, A^{*}\left(x_{1}, x_{2}\right)\right) d F_{1,2}\left(x_{1}, x_{2}\right) \\
& \quad+\int_{\left[x_{2}, 1\right] \times[0,1]} \bar{d}\left(x_{2}, x_{1}, A^{*}\left(x_{1}, x_{2}\right)\right) d F_{1,2}\left(x_{1}, x_{2}\right) \\
& =\int_{[0,1]^{2}} \int_{[0,1]} \min \left\{\left|x_{1}-a\right|,\left|x_{2}-a\right|\right\} d G_{A^{*}\left(x_{1}, x_{2}\right)}(a) d F_{1,2}\left(x_{1}, x_{2}\right) .
\end{aligned}
$$

Therefore, using the inequality in (15), we have that

$$
\begin{aligned}
& \max _{A \in \mathcal{G}_{1}(\mu)} \int_{[0,1]^{2}} \int_{[0,1]} \min \left\{\left|x_{1}-a\right|,\left|x_{2}-a\right|\right\} d G_{A}(a) d F_{1,2}\left(x_{1}, x_{2}\right) \\
& \quad \leq \int_{[0,1]^{2}} \int_{[0,1]} \min \left\{\left|x_{1}-a\right|,\left|x_{2}-a\right|\right\} d G_{A^{*}\left(x_{1}, x_{2}\right)}(a) d F_{1,2}\left(x_{1}, x_{2}\right) .
\end{aligned}
$$

Moreover, since $p^{*}\left(x_{1}, x_{2}\right) \in T\left(x_{1}, x_{2}\right)$, we have that the mean value of $A *\left(x_{1}, x_{2}\right)$ is $\mu$ for all $\left(x_{1}, x_{2}\right) \in[0,1]^{2}$. Therefore, $A *\left(X_{1}, X_{2}\right)$ is also a random variable with mean value $\mu$, that is, $A^{*}\left(X_{1}, X_{2}\right) \in \mathcal{G}_{1}(\mu)$. Thus, the inequality above has to be an equality and Problem (10) can be reformulated as follows:

$$
\begin{aligned}
& \min _{X_{1}, X_{2} \in \mathcal{F}_{1}} \int_{[0,1]^{2}}\left[C \left(x_{1}, x_{2}, p_{1}^{*}\left(x_{1}, x_{2}\right) \mathcal{X}_{S_{1}}\left(x_{1}, x_{2}\right)\right.\right. \\
& \left.\quad+C\left(x_{2}, x_{1}, p_{1}^{*}\left(x_{1}, x_{2}\right)\right) \mathcal{X}_{S_{2}}\left(x_{1}, x_{2}\right)\right] d F_{1,2}\left(x_{1}, x_{2}\right) .
\end{aligned}
$$

Let us define the following function;

$$
L\left(x_{1}, x_{2}\right):=C\left(x_{1}, x_{2}, p_{1}^{*}\left(x_{1}, x_{2}\right) \mathcal{X}_{S_{1}}\left(x_{1}, x_{2}\right)+C\left(x_{2}, x_{1}, p_{1}^{*}\left(x_{1}, x_{2}\right) \mathcal{X}_{S_{2}}\left(x_{1}, x_{2}\right) .\right.\right.
$$


Since, it always holds that,

$$
\min _{X_{1}, X_{2} \in \mathcal{F}_{1}} \int_{[0,1]^{2}} L\left(x_{1}, x_{2}\right) d F_{1,2}\left(x_{1}, x_{2}\right)=\min _{x_{1}, x_{2} \in[0,1]} L\left(x_{1}, x_{2}\right),
$$

then the minimum in (21) is reached by two degenerate random variables. On the other hand, using that the function $L\left(x_{1}, x_{2}\right)$ is defined in disjoint sets, $C\left(x_{1}, x_{2}, p_{1}^{*}\left(x_{1}, x_{2}\right)\right) \mathcal{X}_{S_{1}}\left(x_{1}, x_{2}\right) \geq 0$ and $C\left(x_{2}, x_{1}, p_{1}^{*}\left(x_{1}, x_{2}\right)\right) \mathcal{X}_{S_{2}}\left(x_{1}, x_{2}\right) \geq 0$ (see (19), (20) and Lemma A.5 to justify the non-negativity of these functions), we have that

$$
L\left(x_{1}, x_{2}\right)=\max \left\{C\left(x_{1}, x_{2}, p_{1}^{*}\left(x_{1}, x_{2}\right)\right) \mathcal{X}_{S_{1}}\left(x_{1}, x_{2}\right), C\left(x_{2}, x_{1}, p_{1}^{*}\left(x_{1}, x_{2}\right)\right) \mathcal{X}_{S_{2}}\left(x_{1}, x_{2}\right)\right\} .
$$

Therefore, we can use the same arguments as in the deterministic case (Lemma A.8) in order to obtain that the optimal solutions are the random vectors $\left(X_{1}, X_{2}\right)$ such that:

$$
P\left[\left(X_{1}, X_{2}\right)=\left(\mu^{2}, 2 \mu-\mu^{2}\right)\right]=1 \quad \text { or } \quad P\left[\left(X_{1}, X_{2}\right)=\left(2 \mu-\mu^{2}, \mu^{2}\right)\right]=1 .
$$

In conclusion, Theorem 3.2 proves that it is optimal to park the service units when no hypotheses are made on their c.d.f.'s and only the mean value of the position of the random incident is known.

\section{CONCLUDING REMARKS}

The results in this paper extend other previously known results about the optimal location of one or two service units to situations where no assumptions are made on the probability distribution of the random incident that these service units cover apart from the knowledge of its mean value (whereas all the previous papers require exact knowledge of this distribution). This is accomplished by minimizing the maximum expected response time (whereas the previous results minimize expected distances). In particular, we show that when the only available information is the mean value of the position of the incidents, then the optimal policy is to park the service units at concrete points.

On the other hand, since our goal is to minimize the response time from the service unit to the incident, another interesting problem is to assume the same probability distribution for both the service unit and the incident. Notice that one interpretation of this policy is that we are fixing the location of the service unit at the location of the previous incident. The worst case for this policy is given by

$$
\max _{F \in \mathcal{F}_{1}(\mu)} \int_{[0,1]} \int_{[0,1]}\|x-a\|_{1} d F(a) d F(x)
$$

(assuming that incidents occur independently). Using a similar argument to the one used in the proof of Theorem 2.2, we have that

$$
\max _{F \in \mathcal{F}_{1}(\mu)} \int_{[0,1]} \int_{[0,1]}\|x-a\|_{1} d F(a) d F(x)=\mu+\mu(1-2 \mu) .
$$


Thus, the maximum objective value above is $1 / 2$ and it is achieved by a c.d.f. $F$ with mean value $\mu=1 / 2$. Moreover, by the proof of Corollary 2.1 , we have that the objective value of the worst case for Problem (6) is $\mu+\lambda(1-2 \mu)$. Thus, the best objective value when $\lambda$ varies, is:

$$
\begin{cases}\mu & \text { if } \mu \leq 0.5 \text { and it is achieved at } \lambda^{*}=0 \\ 1-\mu & \text { if } \mu>0.5 \text { and it is achieved at } \lambda^{*}=1 .\end{cases}
$$

Therefore, for a fixed $\mu$, the difference in worst case performance between the approach considered above and the approach of the paper is given by

$$
\left|\left(\lambda^{*}-\mu\right)(1-2 \mu)\right|= \begin{cases}\mu(1-2 \mu) & \text { if } \mu \leq 0.5 \\ (1-\mu)(-1+2 \mu) & \text { if } \mu>0.5\end{cases}
$$

Notice that there is no difference when $\mu \in\{0,0.5,1\}$, but that there is a difference that can be quantified for other choices of $\mu$. We can see that this difference is maximal when $\mu=0.25$ and $\mu=0.75$ which is reasonable because these points are at the maximum distances to the values of $\mu$ where the difference is null.

Finally, we can also study the natural extension of Problem (10) where we consider $k$ service units instead of two. It should be noted that using similar arguments to those used for the case $k=2$, we can obtain that the worst case in the distribution of the incident is given by a random variable taking the values $0, \frac{x_{1}+x_{2}}{2}, \frac{x_{2}+x_{3}}{2}, \ldots, \frac{x_{k-1}+x_{k}}{2}, 1$ (see Eq. (18) for the case when $k=2$ ). However, the complexity of the expressions obtained in the analysis does not allow us to present an explicit formula of the optimal solution of this problem.

\section{APPENDIX}

In this section, we include for the sake of completeness, several results and their proofs which have been used in the paper.

Lemma A.1 Let $G(\cdot)$ be a nondecreasing function such that $G: \mathbb{R} \rightarrow[0, \infty)$. If there exists $M \in \mathbb{R}$ such that

$$
\int_{a}^{b} G(t) d t=M(b-a) \quad \text { with } a, b \in \mathbb{R}
$$

then

$$
I_{G}(z):=\int_{a}^{z} G(t) d t-M(z-a) \leq 0 \quad \forall z \in[a, b] .
$$

Proof: Let $t_{0} \in[a, b]$ be such that $t_{0}=\inf \{t: G(t) \geq M\}$; 
i) If $z<t_{0}$ we have that $G(t)<M \quad \forall t \leq z$ thus, $I_{G}(z) \leq 0$.

ii) If $z \geq t_{0}$ we obtain that,

$$
\begin{aligned}
I_{G}(z) & =\int_{a}^{z} G(t) d t+\int_{z}^{b} G(t) d t-\int_{z}^{b} G(t) d t-M(z-a) \\
& =M(b-a)-\int_{z}^{b} G(t) d t-M(z-a)=M(b-z)-\int_{z}^{b} G(t) d t \\
& \leq M(b-z)-G_{A}(z)(b-z)=(M-G(z)(b-z) \leq 0,
\end{aligned}
$$

where we have used the fact that the function $G(\cdot)$ is nondecreasing. Thus, the lemma is proved.

Lemma A.2 For any $A \in \mathcal{G}_{1}(\mu)$ with c.d.f. $G_{A}(\cdot)$, we have that:

i) $\int_{0}^{1} G_{A}(a) d a=1-\mu$.

ii) $\quad \int_{[0,1]}|\mathrm{x}-\mathrm{a}| \mathrm{dG}_{\mathrm{A}}(\mathrm{a})=2 \int_{0}^{\mathrm{x}} G_{A}(a) d a-x+\mu \quad \forall x \in[0,1]$.

Proof: Denote $D_{G}$ the set of denumerable number of discontinuity points of $\mathrm{G}_{\mathrm{A}}(\cdot)$ in the interval $[0,1]$ union with the set $\{0,1\}$. Applying integration by parts to the interval $\left(x_{i-1}, x_{i}\right)$ where $x_{i-1}$ and $x_{i}$ are two consecutive points of $D_{G}$, we have that

$$
\begin{aligned}
\int_{\left(x_{i-1}, x_{i}\right)} \mathrm{G}_{\mathrm{A}}(a) d a & =\left.a G_{A}(a)\right|_{x_{\mathrm{i}-1}^{+}} ^{x_{\mathrm{i}}^{-}}-\int_{\left(x_{i-1}, x_{i}\right)} a d G_{A}(a) \\
& =\mathrm{x}_{\mathrm{i}}^{-} G_{A}\left(x_{\mathrm{i}}^{-}\right)-x_{i-1}^{+} G_{A}\left(x_{i-1}^{+}\right)-\int_{\left(x_{i-1}, x_{i}\right)} a d G_{A}(a) \\
& =x_{i}\left(G_{A}\left(x_{i}\right)-P\left[A=x_{i}\right]\right)-x_{i-1} G_{A}\left(x_{i-1}\right)-\int_{\left(x_{i-1}, x_{\mathrm{i}}\right)} a d G_{A}(a) \\
& =x_{i} G_{A}\left(x_{i}\right)-x_{i-1} G_{A}\left(x_{i-1}\right)-\int_{\left(x_{i-1}, x_{i}\right)} a d G_{A}(a) .
\end{aligned}
$$

If we sum the equality above for each element of $D_{G}$ we obtain

$$
\begin{aligned}
\sum_{x_{i} \in D_{G}} \int_{\left(x_{i-1}, x_{i}\right)} G_{A}(a) \mathrm{da} & =\sum_{x_{i} \in D_{G}}\left[x_{i} G_{A}\left(x_{i}\right)-x_{i-1} G_{A}\left(x_{i-1}\right)-\int_{\left(x_{i-1}, x_{i}\right]} a d G_{A}(a)\right] \\
& =1-\int_{(0,1]} a d G_{A}(a) .
\end{aligned}
$$


138

\section{Puerto and Rodríguez-Chía}

601

631

Hence, since the integrant in the last expression is null at zero we have that

$$
\int_{0}^{1} G_{A}(a) d a=1-\int_{[0,1]} a d G_{A}(a)=1-\mu .
$$

Now we prove the second assertion. We have the following equalities

$$
\begin{aligned}
\int_{[0,1]}|x-a| d G_{A}(a) & =\int_{[0, x]}(x-a) d G_{A}(a)+\int_{(x, 1]}(a-x) d G_{A}(a) \\
& =x G_{A}(x)-\int_{[0, x]} a d G_{A}(a)+\int_{(x, 1]} a d G_{A}(a)-x\left(1-G_{A}(x)\right) \\
& =x\left(2 G_{A}(x)-1\right)-\int_{[0, x]} a d G_{A}(a)+\mu-\int_{[0, x]} a d G_{A}(a) \\
& =2\left(x G_{A}(x)-\int_{[0, x]} a d G_{A}(a)\right)+\mu-x .
\end{aligned}
$$

Applying integration by parts using the arguments above we have that

$$
\int_{0}^{x} G_{A}(a) d a=x G_{A}(x)-\int_{[0, \mathrm{x}]} a d G_{A}(a),
$$

and the result follows.

Lemma A.3 For any $X \in \mathcal{F}_{1}(\lambda)$ and $A \in \mathcal{G}_{1}(\mu)$ with c.d.f's $F_{X}(\cdot)$ and $G_{A}(\cdot)$, respectively, we have that

$$
\begin{aligned}
\int_{[0,1]} y G_{A}(y) d F_{X}(y)+\int_{[0,1]} y F_{X}(y) d G_{A}(y)= & 1+\sum_{y \in D} y P[X=y] P[A=y] \\
& -\int_{0}^{1} G_{A}(y) F_{X}(y) d y,
\end{aligned}
$$

where $D$ is the set of denumerable number of discontinuity points either of $F_{X}(\cdot)$ or $G_{A}(\cdot)$ (or both) union with the set $\{0,1\}$. 
Proof: Applying integration by parts to the inteval $\left(x_{i-1}, x_{i}\right)$ where $x_{i-1}$ and $x_{i}$ are two consecutive points of $D$, we have the following equalities

$$
\begin{aligned}
& \int\left(x_{i-1}, x_{i}\right) y G_{A}(y) d F_{X}(y)+\int_{\left(x_{i-1}, x_{i}\right)} y F_{X}(y) d G_{A}(y) \\
& =\left.y G_{A}(y) F_{X}(y)\right|_{x_{i-1}^{+}} ^{x_{i}^{-}}-\int_{\left(x_{i-1}, x_{i}\right)} G_{A}(y) F_{X}(y) d y \\
& =x_{i}^{-} G_{A}\left(x_{i}^{-}\right) F_{X}\left(x_{i}^{-}\right)-x_{i-1}^{+} G_{A}\left(x_{i-1}^{+}\right) F_{X}\left(x_{i-1}^{+}\right)-\int_{x_{i-1}}^{x_{i}} G_{A}(y) F_{X}(y) d y \\
& =x_{\mathrm{i}}\left(G_{A}\left(x_{i}\right)-P\left[A=x_{i}\right]\right)\left(F_{X}\left(x_{i}\right)-P\left[X=x_{i}\right]\right)-x_{i-1} G_{A}\left(x_{i-1}\right) F_{X}\left(x_{i-1}\right) \\
& \quad-\int_{x_{i-1}}^{x_{i}} G_{A}(y) F_{X}(y) d y=x_{i} G_{A}\left(x_{i}\right) F_{X}\left(x_{i}\right)-x_{i-1} G_{A}\left(x_{i-1}\right) F_{X}\left(x_{\mathrm{i}-1}\right) \\
& \quad-x_{i} P\left[X=x_{i}\right] G_{A}\left(x_{i}\right)-x_{i} P\left[A=x_{i}\right] F_{X}\left(x_{i}\right)+x_{i} P\left[A=x_{i}\right] P\left[X=x_{i}\right] \\
& \quad-\int_{x_{i-1}}^{x_{i}} G_{A}(y) F_{X}(y) d y .
\end{aligned}
$$

The equality above can be rewritten as

$$
\begin{aligned}
& \int_{\left(x_{i-1}, x_{i}\right]} y G_{A}(y) d F_{X}(y)+\int_{\left(x_{i-1}, x_{i}\right]} y F_{X}(y) d G_{A}(y) \\
& =x_{i} G_{A}\left(x_{i}\right) F_{X}\left(x_{i}\right)-x_{i-1} G_{A}\left(x_{i-1}\right) F_{X}\left(x_{i-1}\right) \\
& \quad+x_{i} P\left[A=x_{i}\right] P\left[X=x_{i}\right]-\int_{x_{i-1}}^{x_{i}} G_{A}(y) F_{X}(y) d y .
\end{aligned}
$$

If we sum the expression above for each element of $D$ we have

$$
\begin{aligned}
\sum_{x_{i} \in D} & {\left[\int_{\left(x_{i-1}, x_{i}\right]} y G_{A}(y) d F_{X}(y)+\int_{\left(x_{i-1}, x_{i}\right]} y F_{X}(y) d G_{A}(y)\right] } \\
= & \sum_{x_{i} \in D}\left[x_{i} G_{A}\left(x_{i}\right) F_{X}\left(x_{i}\right)-x_{i-1} G_{A}\left(x_{i-1}\right) F_{X}\left(x_{i-1}\right)-+x_{i} P\left[A=x_{i}\right] P\left[X=x_{i}\right]\right. \\
& \left.-\int_{x_{i-1}}^{x_{i}} G_{A}(y) F_{X}(y) d y\right]=1+\sum_{x_{i} \in D} x_{i} P\left[A=x_{i}\right] P\left[X=x_{i}\right]-\int_{0}^{1} G_{A}(y) F_{X}(y) d y .
\end{aligned}
$$




\section{Puerto and Rodríguez-Chía}

Since, at zero the integrants of the first part of the equalities above are null, we have that

$$
\begin{aligned}
\int_{[0,1]} y G_{A}(y) d F_{X}(y)+\int_{[0,1]} y F_{X}(y) d G_{A}(y)= & +\sum_{x_{i} \in D} x_{i} P\left[A=x_{i}\right] P\left[X=x_{i}\right] \\
& -\int_{0}^{1} G_{A}(y) F_{X}(y) d y,
\end{aligned}
$$

and the result follows.

Lemma A.4 For any $X \in \mathcal{F}_{1}(\lambda)$ and $\mathrm{A} \in \mathcal{G}_{1}(\mu)$ with c.d.f's $F_{X}(\cdot)$ and $G_{A}(\cdot)$, respectively, we have that

$$
\int_{[0,1]} \int_{[0,1]}|x-a| d G_{A}(a) d F_{X}(x)=2\left(1-\int_{0}^{1} G_{A}(y) F_{X}(y) d y\right)-\lambda-\mu .
$$

Proof: We have that

$$
\begin{aligned}
& \int_{[0,1]} \int_{[0,1]}|\mathrm{x}-\mathrm{a}| d G_{A}(a) d F_{X}(x) \\
&=\int_{[0,1]} \int_{[0, x]} x d G_{A}(a) d F_{X}(x)-\int_{[0,1]} \int_{[0, x]} a d G_{A}(a) d F_{X}(x) \\
&+\int_{[0,1]} \int_{(x, 1]} a d G_{A}(a) d F_{X}(x)-\int_{[0,1]} \int_{(x, 1]} x d G_{A}(a) d F_{X}(x) \\
&= \int_{[0,1]} x G_{A}(x) d F_{X}(x)-\int_{[0,1]} \int_{[0, x]} a d G_{A}(a) d F_{X}(x) \\
&+\int_{[0,1]} \int_{(x, 1]} a d G_{A}(a) d F_{X}(x)-\int_{[0,1]} x\left(1-G_{A}(x)\right) d F_{X}(x) \\
&= 2 \int_{[0,1]} x G_{A}(x) d F_{X}(x)-\lambda-\int_{[0,1]} \int_{[0, x]} a d G_{A}(a) d F_{X}(x) \\
&+\int_{[0,1]}\left(\mu-\int_{[0, x]} a d G_{A}(a)\right) d F_{X}(x)=2 \int_{[0,1]} x G_{A}(x) d F_{X}(x) \\
&-2 \int_{[0,1]} \int_{[0, x]} a d G_{A}(a) d F_{X}(x)-\lambda+\mu=2 \int_{[0,1]} x G_{A}(x) d F_{X}(x) \\
&-2 \int_{[0,1]} a \int_{[a, 1]} d F_{X}(x) d G_{A}(a)-\lambda+\mu=2 \int_{[0,1]} x G_{A}(x) d F_{X}(x) \\
&- 2 \int_{[0,1]} a \int_{(a, 1]} d F_{X}(x) d G_{A}(a)-2 \int_{[0,1]} a P[X=a] d G_{A}(a)-\lambda+\mu .
\end{aligned}
$$


Denote by $D$ the denumerable number of discontinuity points of $F_{X}$ or $G_{A}$ union with $\{0,1\}$. Then, we rewrite the expression above as

$$
\begin{aligned}
& 2 \int_{[0,1]} x G_{A}(x) d F_{X}(x)-2 \mu+2 \int_{[0,1]} a F_{X}(a) d G_{A}(a)-2 \sum_{y \in D} y P[X=y] P[A=y]-\lambda+\mu \\
& \quad=2\left(\int_{[0,1]} y G_{A}(y) d F_{X}(y)+\int_{[0,1]} y F_{X}(y) d G_{A}(y)\right)-2 \sum_{y \in D} y P[X=y] P[A=y]-\lambda-\mu .
\end{aligned}
$$

Now, using Lemma A.3 we have that

$$
(22)=2\left(1-\int_{0}^{1} G_{A}(y) F_{X}(\mathrm{y}) d y\right)-\lambda-\mu,
$$

and the result follows.

Lemma A.5 For any $A \in \mathcal{G}_{1}(\mu)$ with c.d.f. $G_{A}(\cdot)$, the function $\bar{d}$ defined in (12) admits the following representation.

$$
\bar{d}\left(x_{1}, x_{2}, A\right)=\int_{[0,1]} \min \left\{\left|x_{1}-a\right|,\left|x_{2}-a\right|\right\} d G_{A}(a) \quad \forall x_{1} \leq x_{2} \in[0,1] .
$$

Proof: We have the following equalities:

$$
\begin{aligned}
\int_{[0,1]} \min \left\{\left|x_{1}-a\right|,\left|x_{2}-a\right|\right\} d G_{A}(a)= & \int_{\left[0, \frac{x_{1}+x_{2}}{2}\right]}\left|x_{1}-a\right| d G_{A}(a)+\int_{\left.\frac{x_{1}+x_{2}}{2}, 1\right]}\left|x_{2}-a\right| d G_{A}(a) \\
= & \int_{\left[0, x_{1}\right]}\left(x_{1}-a\right) d G_{A}(a)+\int_{\left(x_{1}, \frac{x_{1}+x_{2}}{2}\right]}\left(a-x_{1}\right) d G_{A}(a) \\
& +\int_{\left.\frac{x_{1}+x_{2}}{2}, x_{2}\right]}\left(x_{2}-a\right) d G_{A}(a)+\int_{\left(x_{2}, 1\right]}\left(a-x_{2}\right) d G_{A}(a) \\
= & 2 x_{1} G_{A}\left(x_{1}\right)-G_{A}\left(\frac{x_{1}+x_{2}}{2}\right)\left(x_{1}+x_{2}\right)+2 x_{2} G_{A}\left(x_{2}\right) \\
& -x_{2}-\int_{\left[0, x_{1}\right]} a d G_{A}(a)+\int_{\left(x_{1}, \frac{x_{1}+x_{2}}{2}\right]} a d G_{A}(a) \\
& -\int_{\left(\frac{x_{1}+x_{2}}{2}, x_{2}\right]} a d G_{A}(a)+\int_{\left(x_{2}, 1\right]} a d G_{A}(a) \\
= & 2\left(x_{1} G_{A}\left(x_{1}\right)-\int_{\left[0, x_{1}\right]} a d G_{A}(a)+x_{2} G_{A}\left(x_{2}\right)\right.
\end{aligned}
$$




\section{Puerto and Rodríguez-Chía}

$$
\begin{aligned}
& \left.-G_{A}\left(\frac{x_{1}+x_{2}}{2}\right) \frac{x_{1}+x_{2}}{2}-\int_{\frac{\left(\frac{x_{1}+x_{2}}{2}, x_{2}\right]}{}} a d G_{A}(a)\right) \\
& +\mu-x_{2}=2\left(\int_{0}^{x_{1}} G_{A}(a) d a+\int_{\frac{x_{1}+x_{2}}{2}}^{x_{2}} G_{A}(a) d a\right) \\
& +\mu-x_{2}=\bar{d}\left(x_{1}, x_{2}, A\right),
\end{aligned}
$$

and the result is proved.

Lemma A.6 For each random variable $A \in \mathcal{G}_{1}(\mu)$ with distribution function $G_{A}(\cdot)$ and each $0 \leq x_{1} \leq x_{2} \leq 1$, there exists a discrete random variable $\bar{A} \in \mathcal{G}_{1}(\mu)$ defined by

$$
P[\bar{A}=a]=\left\{\begin{array}{lll}
p_{0} & \text { if } & a=0 \\
p_{1} & \text { if } & a=\frac{x_{1}+x_{2}}{2} \\
p_{2} & \text { if } & a=1,
\end{array}\right.
$$

where $\left(p_{0}, p_{1}, p_{2}\right)$ satisfies that

$$
\begin{aligned}
& p_{0}+p_{1}+p_{2}=1 \\
& \bar{d}\left(x_{1}, x_{2}, A\right) \leq \bar{d}\left(x_{1}, x_{2}, \bar{A}\right) \\
& \int_{0}^{\frac{x_{1}+x_{2}}{2}} G_{A}(a) d a=p_{0} \frac{x_{1}+x_{2}}{2} \\
& \int_{\frac{x_{1}+x_{2}}{2}}^{1} G_{A}(a) d a=\left(p_{0}+p_{1}\right)\left(1-\frac{x_{1}+x_{2}}{2}\right) .
\end{aligned}
$$

Remark A.1 It should be noted that from this result and part i) of Lemma A.2, one obtains that for each $0 \leq x_{1} \leq x_{2} \leq 1$, the $\max _{A \in \mathcal{G}_{1}(\mu)} \bar{d}\left(x_{1}, x_{2}, A\right)$ is attained in a discrete random variable with mean value $\mu$ and defined only on the values $0, \frac{x_{1}+x_{2}}{2}$ and 1 . Moreover, $p=\left(p_{0}, p_{1}, p_{2}\right) \in T\left(x_{1}, x_{2}\right)$ (where $T$ was defined in (14)).

Proof: First, we note that $\bar{A} \in \mathcal{G}_{1}(\mu)$, see Remark A.1.

Second, in order to complete the proof of the lemma it suffices to prove:

i) $\int_{0}^{x_{1}} G_{A}(a) d a-p_{0} x_{1} \leq 0$

ii) $\int_{\frac{x_{1}+x_{2}}{2}}^{x_{2}} G_{A}(a) d a-\left(p_{0}+p_{1}\right)\left(x_{2}-\frac{x_{1}+x_{2}}{2}\right) \leq 0$.

To this end, we apply Lemma A..1. Since (23) and (24) hold and $G_{A}(\cdot)$ is a probability distribution function, we are under hypotheses of Lemma A.1 and thus, i) and ii) are proved. 
Lemma A.7 If $x_{1}, x_{2} \in[0,1], x_{1} \leq x_{2}$, then

$$
\max _{A \in \mathcal{G}_{1}(\mu)} \bar{d}\left(x_{1}, x_{2}, A\right)=\max _{p \in T\left(x_{1}, x_{2}\right)} C\left(x_{1}, x_{2}, p_{1}\right),
$$

where $\bar{d}, C$, and $T$ were defined in (12), (13), and (14), respectively.

Proof: We have, by the definition of $\bar{d}$ in (12) and Lemma A.6, that;

$$
\begin{aligned}
\max _{A \in \mathcal{G}_{1}(\mu)} \bar{d}\left(x_{1}, x_{2}, A\right) & =\max _{A \in \mathcal{G}_{1}(\mu)} 2\left(\int_{0}^{x_{1}} G_{A}(a) d a+\int_{\frac{x_{1}+x_{2}}{2}}^{x_{2}} G_{A}(a) d a\right)+\mu-x_{2} \\
& =\max _{p \in T\left(x_{1}, x_{2}\right)} 2\left(p_{0} x_{1}+\left(p_{0}+p_{1}\right)\left(x_{2}-\frac{x_{1}+x_{2}}{2}\right)\right)+\mu-x_{2} .
\end{aligned}
$$

Using that $p \in T\left(x_{1}, x_{2}\right)$, (i.e., $p_{0}+p_{1}+p_{2}=1$ and $\frac{x_{1}+x_{2}}{2} p_{1}+p_{2}=\mu$ ) we have that

$$
\begin{aligned}
& \max _{p \in T\left(x_{1}, x_{2}\right)} 2\left(p_{0} x_{1}+\left(p_{0}+p_{1}\right)\left(x_{2}-\frac{x_{1}+x_{2}}{2}\right)\right)+\mu-x_{2} \\
& \underset{p \in T\left(x_{1}, x_{2}\right)}{=\max _{1}} \mu-x_{2}+2\left(\left(1-\mu+p_{1}\left(\frac{x_{1}+x_{2}}{2}-1\right)\right) x_{1}\right. \\
& \left.+\left(1-\mu+p_{1} \frac{x_{1}+x_{2}}{2}\right)\left(x_{2}-\frac{x_{1}+x_{2}}{2}\right)\right) \\
& =\max _{p \in T\left(x_{1}, x_{2}\right)} \mu-x_{2}+2\left((1-\mu) \frac{x_{1}+x_{2}}{2}-p_{1}\left(x_{1}-\left(\frac{x_{1}+x_{2}}{2}\right)^{2}\right)\right) \\
& =\max _{p \in T\left(x_{1}, x_{2}\right)} C\left(x_{1}, x_{2}, p_{1}\right),
\end{aligned}
$$

which proves the result.

Lemma A.8 The optimal solution for the problem

$$
\min _{0 \leq x_{1} \leq x_{2} \leq 1} \max _{p \in T\left(x_{1}, x_{2}\right)} C\left(x_{1}, x_{2}, p_{1}\right)
$$

is $x_{1}=\mu^{2}$ and $x_{2}=2 \mu-\mu^{2}$, where $C$ and $T$ were defined in (13) and (14), respectively.

Proof: Since $C\left(x_{1}, x_{2}, p_{1}\right)$ is linear with respect to $p_{1}$, we analyze the cases where the coefficient that multiplies $p_{1}$ is positive or negative separately. 


\section{Puerto and Rodríguez-Chía}

919

920

Case 1: $x_{1} \geq\left(\frac{x_{1}+x_{2}}{2}\right)^{2}$

In this case, the function $C\left(x_{1}, x_{2}, p_{1}\right)$ is decreasing in $p_{1}$, thus the maximum is reached at $p_{1}=0$. That means that $p_{0}=1-\mu$ and $p_{2}=\mu$. Therefore, the expression that we have to consider is the following:

$$
\begin{aligned}
& \min _{0 \leq x_{1} \leq x_{2} \leq 1} C_{1}\left(x_{1}, x_{2}\right):=2\left((1-\mu) \frac{x_{1}+x_{2}}{2}\right)-x_{2}+\mu \\
& \text { s.t. } \quad x_{1} \geq\left(\frac{x_{1}+x_{2}}{2}\right)^{2} .
\end{aligned}
$$

It is clear that:

$$
\frac{\partial C_{1}\left(x_{1}, x_{2}\right)}{\partial x_{1}}=1-\mu \geq 0 .
$$

Since the function $C_{1}\left(x_{1}, x_{2}\right)$ is increasing in $x_{1}$ and $x_{1} \geq\left(\frac{x_{1}+x_{2}}{2}\right)^{2}$ we have that

$$
C_{1}\left(x_{1}, x_{2}\right) \geq C_{1}\left(t, x_{2}\right),
$$

where $t=\left(\frac{t+x_{2}}{2}\right)^{2}$. Thus, since $x_{2} \geq 0$, this implies that $x_{2}=2 \sqrt{t}-t$ (notice that $2 \sqrt{t}-$ $t \geq t$ for all $t \geq 0$ ).Therefore, solving (26) is equivalent to solving the following problem;

$$
\min _{x_{1} \in[0,1]} 2(1-\mu) \sqrt{x_{1}}-2 \sqrt{x_{1}}+x_{1}+\mu=\min _{x_{1} \in[0,1]} x_{1}+\left(1-2 \sqrt{x_{1}}\right) \mu,
$$

and this problem reaches its minimum at the point $x_{1}=\mu^{2}$. Hence, $x_{2}=2 \mu-\mu^{2}$ and the minimum objective value is $\mu-\mu^{2}$.

Case 2: $x_{1} \leq\left(\frac{x_{1}+x_{2}}{2}\right)^{2}$

Notice that in Case 1, we have already studied the points $(0,0)$ and $(1,1)$. Therefore, in what follows, we can assume without loss of generality that $\left(x_{1}, x_{2}\right)$ is neither $(0,0)$ nor $(1,1)$. In this case, the function $C\left(x_{1}, x_{2}, p_{1}\right)$ is increasing in $p_{1}$. Since $p \in T\left(x_{1}, x_{2}\right)$ we have that $p_{0}=1-\mu+p_{1}\left(\frac{x_{1}+x_{2}}{2}-1\right), p_{2}=\mu-p_{1} \frac{x_{1}+x_{2}}{2}, 0 \leq p_{0} \leq 1$ and $0 \leq p_{2} \leq 1$ then, we have that,

a) $0 \leq(1-\mu)-p_{1}\left(1-\frac{x_{1}+x_{2}}{2}\right) \leq 1$, that is, $-\frac{\mu}{1-\frac{x_{1}+x_{2}}{2}} \leq p_{1} \leq \frac{1-\mu}{1-\frac{x_{1}+x_{2}}{2}}$ if $\left(x_{1}, x_{2}\right) \neq$ $(1,1)$.

b) $0 \leq \mu-p_{1} \frac{x_{1}+x_{2}}{2} \leq 1$, that is, $-\frac{1-\mu}{\frac{x_{1}+x_{2}}{2}} \leq p_{1} \leq \frac{\mu}{\frac{x_{1}+x_{2}}{2}}$ if $\left(x_{1}, x_{2}\right) \neq(0,0)$.

Using that $p_{1} \geq 0,-\frac{\mu}{1-\frac{x_{1}+x_{2}}{2}} \leq 0$ and $-\frac{1-\mu}{\frac{x_{1}+x_{2}}{2}} \leq 0$ the previous conditions reduce to;

a) $p_{1} \leq \frac{1-\mu}{1-\frac{x_{1}+x_{2}}{2}}$

b) $p_{1} \leq \frac{\mu}{\frac{x_{1}+x_{2}}{2}}$.

Hence, $p_{1} \leq \min \left\{\frac{1-\mu}{1-\frac{x_{1}+x_{2}}{2}}, \frac{\mu}{\frac{x_{1}+x_{2}}{2}}\right\}$ and to study this minimum we distinguish two cases; 
- Case 2.1: If $\frac{x_{1}+x_{2}}{2} \geq \mu$ then $\frac{1-\mu}{1-\frac{x_{1}+x_{2}}{2}} \geq \frac{\mu}{\frac{x_{1}+x_{2}}{2}}$, thus $p_{1} \leq \frac{\mu}{\frac{x_{1}+x_{2}}{2}}$.

- Case 2.2: If $\frac{x_{1}+x_{2}}{2} \leq \mu$ then $\frac{1-\mu}{1-\frac{x_{1}+x_{2}}{2}} \leq \frac{\mu}{\frac{x_{1}+x_{2}}{2}}$, thus, $p_{1} \leq \frac{1-\mu}{1-\frac{x_{1}+x_{2}}{2}}$.

Since the function $C\left(x_{1}, x_{2}, p_{1}\right)$ is increasing in $p_{1}$, in Case 2.1. its maximum in $p_{1}$ is reached at $p_{1}=\frac{\mu}{\underline{x_{1}+x_{2}}}$ and in Case 2.2 at $p_{1}=\frac{1-\mu}{1-\frac{x_{1}+x_{2}}{2}}$.

Hence, to find the maximum of the function $C\left(x_{1}, x_{2}, p_{1}\right)$ we have the following two cases:

- $\quad$ Case 2.1: $p_{1}=\frac{\mu}{\frac{x_{1}+x_{2}}{2}}$.

- Case 2.2: $p_{1}=\frac{1-\mu}{1-\frac{x_{1}+x_{2}}{2}}$.

Case 2.1: $\quad p_{1}=\frac{\mu}{x_{1}+x_{2}}$

In this case, Problem (25) reduces to the following optimization problem

$$
\begin{gathered}
\min _{0 \leq x_{1} \leq x_{2} \leq 1} C_{2}\left(x_{1}, x_{2}\right):=x_{1}\left(1-\frac{2 \mu}{\frac{x_{1}+x_{2}}{2}}\right)+\mu \\
\text { s.t. }: x_{1} \leq\left(\frac{x_{1}+x_{2}}{2}\right)^{2} \mu \leq \frac{x_{1}+x_{2}}{2} .
\end{gathered}
$$

We obtain that

$$
\frac{\partial C_{2}\left(x_{1}, x_{2}\right)}{\partial x_{2}}=\mu \frac{x_{1}}{\left(\frac{x_{1}+x_{2}}{2}\right)^{2}} \geq 0 .
$$

Therefore, $C_{2}\left(x_{1}, x_{2}\right)$ is a increasing function in $x_{2}$. Since in this case, $\left(x_{1}, x_{2}\right)$ satisfies that $x_{2} \geq 2 \mu-x_{1}$ and $x_{2} \geq 2 \sqrt{x_{1}}-x_{1}$ we have that

$$
C_{2}\left(x_{1}, x_{2}\right) \geq C_{2}\left(x_{1}, t\right)
$$

where

- $t=2 \mu-x_{1}$ if $x_{1} \leq \mu^{2}$

- $t=2 \sqrt{x_{1}}-x_{1}$ if $x_{1} \geq \mu^{2}$.

Thus,

a) If $x_{1} \leq \mu^{2}$ we have that

$$
\min _{x_{1}, x_{2} \in[0,1]} C_{2}\left(x_{1}, x_{2}\right)=\min _{x_{1} \in[0,1]} \mu-x_{1} .
$$

b) If $x_{1} \geq \mu^{2}$ we have that

$$
\min _{x_{1}, x_{2} \in[0,1]} C_{2}\left(x_{1}, x_{2}\right)=\min _{x_{1} \in[0,1]} x_{1}\left(1-\frac{2 \mu}{\sqrt{x_{1}}}\right)+\mu .
$$


146

\section{Puerto and Rodríguez-Chía}

970

971

972

973

974

975

976

977

978

979

980

981

982

983

984

985

986

987

988

989

990

991

992

993

994

995

996

997

998

999

1000

1001

1002

1003

1004

1005

1006

1007

1008

1009

1010

1011

1012

Both cases give us the same optimal solution $x_{1}=\mu^{2}$ and $x_{2}=2 \mu-\mu^{2}$ and its objective value is $\mu-\mu^{2}$.

Case 2.2: $\quad p_{1}=\frac{1-\mu}{1-\frac{x_{1}+x_{2}}{2}}$

In this case, Problem (25) reduces to the following optimization problem

$$
\begin{aligned}
& \min _{0 \leq x_{1} \leq x_{2} \leq 1} C_{3}\left(x_{1}, x_{2}\right): \min _{x_{1}, x_{2} \in[0,1]} x_{2}\left(\frac{1-\mu}{1-\frac{x_{1}+x_{2}}{2}}-1\right)-x_{1} \frac{1-\mu}{1-\frac{x_{1}+x_{2}}{2}}+\mu \\
& \text { s.t. } x_{1} \leq\left(\frac{x_{1}+x_{2}}{2}\right)^{2} \\
& \mu \geq \frac{x_{1}+x_{2}}{2} .
\end{aligned}
$$

We obtain that,

$$
\frac{\partial C_{3}\left(x_{1}, x_{2}\right)}{\partial x_{1}}=\frac{(1-\mu)\left(x_{2}-1\right)}{\left(1-\frac{x_{1}+x_{2}}{2}\right)^{2}} \leq 0 .
$$

That means that $C_{3}\left(x_{1}, x_{2}\right)$ is a decreasing function in $x_{1}$. Since, in this case, $\left(x_{1}, x_{2}\right)$ satisfies that $x_{1} \leq\left(\frac{x_{1}+x_{2}}{2}\right)^{2}$ and $\mu \geq \frac{x_{1}+x_{2}}{2}$, we have that $x_{1} \leq \mu^{2}$ then

$$
C_{3}\left(x_{1}, x_{2}\right) \geq C_{3}\left(\mu^{2}, x_{2}\right) .
$$

Thus, taking $x_{1}=\mu^{2}$ we have that $\mu \geq \frac{\mu^{2}+x_{2}}{2}$ and $\mu^{2} \leq\left(\frac{\mu^{2}+x_{2}}{2}\right)^{2}$, that is, $x_{2} \leq 2 \mu-\mu^{2}$ and $x_{2} \geq 2 \mu-\mu^{2}$. (Notice that we do not have to consider the other solution $x_{2} \geq$ $-2 \mu-\mu^{2}$ since $-2 \mu-\mu^{2} \leq 0$ and $x_{2} \geq 0$ ). Therefore, $x_{2}=2 \mu-\mu^{2}$.

Since, all the cases give us the same optimal solution, the optimal solution to Problem (25) is $x_{1}=\mu^{2}$ and $x_{2}=2 \mu-\mu^{2}$.

\section{ACKNOWLEDGMENTS}

The authors would like to thank the suggestions made by two anonymous referees and the editor who have improved the readability and the final presentation of the paper. This research has been partially financed by Spanish research grants BFM2001-2378 and BFM2001-4028.

\section{REFERENCES}

1. Anderson, L.R.; Fontenot, R.A. Optimal positioning of service units along a coordinate line. Transportation Sci. 1992, 26 (4), 346-351. 
2. Carrizosa, E.; Muñoz-Márquez, M.; Puerto, J. A note on the optimal positioning of service units. Oper. Res. 1998, 46 (1), 155-156.

3. Flury, B.A. Principal points. Biometrika 1990, 77 (1), 33-41.

4. Gallego, G. A minmax distribution free procedure for the $(Q, R)$ inventory model. Oper. Res. Lett. 1992, 11, 55-60.

5. Larson, R.; Odoni, A. Urban Operations Research; Prentice-Hall Englewood Cliffs: New Jersey, 1981.

6. Levine, A. A patrol problem. Math. Mag. 1986, 59, 159-166.

7. De Palma, A.; Liu, Q.; Thisse, J.F. Optimal location on a line with random utilities. Transportation Sci. 1994, 28 (1), 63-69.

8. Puerto, J.; Fernández, F.R. Pareto-optimality in classical inventory problems. Naval Res. Logistic 1998, 45, 83-98.

9. Smith, D.K. Police patrol policies on motorways with unequal patrol lengths. J. Oper. Res. Soc. 1997, 48, 996-1000.

10. Vickson, R.G.; Gerchak, Y.; Rotem, D. Optimal positioning of read/write head in mirrored disks. Location Sci. 1995, 3 (2), 125-132.

Received April 20, 1999

Revised April 18, 2000

Accepted October 1, 2002 